IJMMS 27:11 (2001) 689-694

PII. S016117120101078X

http://ijmms.hindawi.com

(c) Hindawi Publishing Corp.

\title{
FLETT'S MEAN VALUE THEOREM IN TOPOLOGICAL VECTOR SPACES
}

\section{ROBERT C. POWERS, THOMAS RIEDEL, and PRASANNA K. SAHOO}

(Received 23 August 2000 and in revised form 22 February 2001)

\begin{abstract}
We prove some generalizations of Flett's mean value theorem for a class of Gateaux differentiable functions $f: X \rightarrow Y$, where $X$ and $Y$ are topological vector spaces.

2000 Mathematics Subject Classification. 58C20, 26E20, 46G05.
\end{abstract}

1. Introduction. In 1958 , Flett proved that if $f:[a, b] \rightarrow \mathbb{R}$ is differentiable on $[a, b]$ and satisfies $f^{\prime}(a)=f^{\prime}(b)$, then there exists $\eta$ in the open interval $(a, b)$ such that $f(\eta)-f(a)=(\eta-a) f^{\prime}(\eta)$ [3]. Flett's conclusion implies that the tangent at $(\eta, f(\eta))$ passes through the point $(a, f(a))$. A recent article by Khan [4], which generalizes the Mean Value Theorem to the context of topological vector spaces, stimulated us to see if there was a similar generalization of Flett's theorem. It turns out that such a generalization does exist. However, even more can be done.

This article focuses on three distinct generalizations of Flett's theorem. First, we drop the endpoint condition $f^{\prime}(a)=f^{\prime}(b)$. Second, we consider the case where $f$ is not differentiable at a finite number of points. Third, we drop differentiability. As expected, the conclusion at each step is weaker than the previous conclusion. These generalizations are given in Theorem 2.1. We then place these results in a topological vector space setting replacing ordinary differentiability with Gateaux differentiability.

2. Results. Let $f:[a, b] \rightarrow \mathbb{R}$ be continuous on $[a, b]$. Following [2] we will say that the graph of $f$ intersects its chord in the extended sense if either there is a number $c \in(a, b)$ such that

$$
(f(c)-f(a))(b-a)=(c-a)(f(b)-f(a))
$$

or

$$
\lim _{x \rightarrow a^{+}} \frac{f(x)-f(a)}{x-a}=\frac{f(b)-f(a)}{b-a}
$$

We now state some interesting generalizations of Flett's theorem.

THEOREM 2.1. Let $f:[a, b] \rightarrow \mathbb{R}$ be continuous on $[a, b]$. Let $J=\{x \in[a, b]: f$ is not differentiable at $x\}$ and set $j=|J|$. For each $x \in(a, b] \backslash J$, let

$$
F \ell(x)=\frac{1}{(x-a)^{2}}\left[(x-a) f^{\prime}(x)-(f(x)-f(a))\right] .
$$


(1) If $j=0$, then there exists a point $\eta \in(a, b)$ such that

$$
F \ell(\eta)=\frac{1}{2} \frac{f^{\prime}(b)-f^{\prime}(a)}{b-a} .
$$

(2) If $j \leq n$ for some nonnegative integer $n$ and $a \notin J$, then there exist $n+1$ points $\eta_{1}, \eta_{2}, \ldots, \eta_{n+1} \in(a, b)$ and $n+1$ positive numbers $\alpha_{1}, \alpha_{2}, \ldots, \alpha_{n+1}$ such that

$$
\begin{gathered}
\alpha_{1}+\alpha_{2}+\cdots+\alpha_{n+1}=1, \\
\sum_{i=1}^{n+1} \alpha_{i} F \ell\left(\eta_{i}\right)=\frac{1}{(b-a)^{2}}\left[(f(b)-f(a))-(b-a) f^{\prime}(a)\right] .
\end{gathered}
$$

(3) If $j$ is unbounded and the graph of $f$ intersects its chord in the extended case, then there exist $c$ in $(a, b)$, and two positive numbers $\delta_{1}, \delta_{2}$ such that either

$$
F \ell_{1}(c, h) \leq 0 \leq F \ell_{2}(c, k)
$$

or

$$
F \ell_{2}(c, k) \leq 0 \leq F \ell_{1}(c, h)
$$

holds for $0<h \leq \delta_{1}$ and $0<k \leq \delta_{2}$ where

$$
\begin{aligned}
& F \ell_{1}(c, h)=\left[(c-a) \frac{f(c)-f(c-h)}{h}-(f(c)-f(a))\right], \\
& F \ell_{2}(c, k)=\left[(c-a) \frac{f(c+k)-f(c)}{k}-(f(c)-f(a))\right] .
\end{aligned}
$$

Some remarks are in order. Flett's original result is the case where $F \ell(\eta)=0$. In item (2) we note that if $f^{\prime}(a)=(f(b)-f(a)) /(b-a)$, that is, the second condition for the graph of $f$ intersecting its chord in the extended sense holds, then $\sum_{i=1}^{n+1} \alpha_{i} F \ell\left(\eta_{i}\right)=0$. If, in item (3), $f$ is differentiable at $c$, then

$$
\lim _{h \rightarrow 0^{+}} \frac{F \ell_{1}(c, h)}{(c-a)^{2}}=\lim _{k \rightarrow 0^{+}} \frac{F \ell_{2}(c, k)}{(c-a)^{2}}=F \ell(c) .
$$

A proof of item (1) can be found in [1] and a proof of item (3) can be found in [2]. In order to prove item (2) we need the following lemma.

LEMMA 2.2. Let $g:[a, b] \rightarrow \mathbb{R}$ be continuous on $[a, b]$ and differentiable on the open interval $(a, b)$ except possibly at a finite number, $n$, of points. Then there exist $n+1$ points $\eta_{1}, \eta_{2}, \ldots, \eta_{n+1} \in(a, b)$ and $n+1$ positive numbers $\alpha_{1}, \alpha_{2}, \ldots, \alpha_{n+1}$ such that

$$
\begin{gathered}
\alpha_{1}+\alpha_{2}+\cdots+\alpha_{n+1}=1, \\
g(b)-g(a)=(b-a) \sum_{i=1}^{n+1} \alpha_{i} g^{\prime}\left(\eta_{i}\right) .
\end{gathered}
$$

Notice that Lemma 2.2 is a generalization of the mean value theorem. A proof of Lemma 2.2 can be found in [5]. 
Proof OF (2). Consider the function $g:[a, b] \rightarrow \mathbb{R}$ defined by

$$
g(x)= \begin{cases}\frac{f(x)-f(a)}{x-a} & \text { if } x \in(a, b], \\ f^{\prime}(a) & \text { if } x=a .\end{cases}
$$

Note that $g$ is continuous on $[a, b]$ and satisfies the hypotheses of Lemma 2.2. Further

$$
g^{\prime}(x)=-\frac{f(x)-f(a)}{(x-a)^{2}}+\frac{f^{\prime}(x)}{x-a}
$$

where $f^{\prime}$ exists; this implies

$$
g^{\prime}(x)=-\frac{g(x)}{x-a}+\frac{f^{\prime}(x)}{x-a}
$$

Applying Lemma 2.2, we get

$$
g(b)-g(a)=(b-a) \sum_{i=1}^{n+1} \alpha_{i} g^{\prime}\left(\eta_{i}\right) .
$$

After simplifying the previous expression the result follows.

Our main goal is to place Theorem 2.1 in the context of topological vector spaces. However, an exact analogue of Theorem 2.1 for vector-valued functions is not true. For example, to see why item (1) fails consider the function $f:[0,2 \pi] \rightarrow \mathbb{R}^{2}$ defined by

$$
f(x)=(\cos (x), \sin (x)-x)
$$

for all $x \in[0,2 \pi]$. Then

$$
f^{\prime}(x)=(-\sin (x), \cos (x)-1)
$$

Therefore, we have $f^{\prime}(0)=(0,0)=f^{\prime}(2 \pi)$, that is, the derivatives of $f$ at the endpoints of the closed interval $[0,2 \pi]$ are equal. Nevertheless, it is not hard to show that the equation

$$
f(\eta)-f(0)=\eta f^{\prime}(\eta)
$$

has no solution in $(0,2 \pi)$.

In the sequel, we will let $X$ and $Y$ be Hausdorff topological vector spaces over the field $\mathbb{R}$ of real numbers, and $A \subset X$ be an open set. Furthermore, we assume that $Y$ has a continuous dual $Y^{*}$. A function $f: A \rightarrow Y$ is said to be Gateaux differentiable at $x_{0} \in A$ if there exists a mapping from $X$ into $Y$, denoted by $f^{\prime}\left(x_{0}\right)$, such that, given any $z \in X$ and a balanced neighborhood $V$ of 0 in $Y$, there exists a $\delta>0$ satisfying

$$
\frac{f\left(x_{0}+t z\right)-f\left(x_{0}\right)}{t}-f^{\prime}\left(x_{0}\right)(z) \in V
$$


whenever $0<|t|<\delta ; f^{\prime}\left(x_{0}\right)$ is called the Gateaux derivative of $f$ at $x_{0}$ and we write

$$
f^{\prime}\left(x_{0}\right)(z)=\lim _{t \rightarrow 0} \frac{f\left(x_{0}+t z\right)-f\left(x_{0}\right)}{t}, \quad z \in X .
$$

Note that a Gateaux differentiable function need not be continuous. For example, the function $f: \mathbb{R}^{2} \rightarrow \mathbb{R}$, given by

$$
f(z)= \begin{cases}\frac{u v^{2}}{u^{2}+v^{4}} & \text { if } z=(u, v) \neq(0,0), \\ 0 & \text { if } z=(u, v)=(0,0)\end{cases}
$$

is not continuous at $(0,0)$ although $f^{\prime}((0,0))(z)$ exists.

Let $[a, b] \subset A$. A function $f:[a, b] \rightarrow Y$ is said to intersect its chord in the extended sense if either there is an $l$ in $(0,1)$ and a $u \in Y^{*}$ such that

$$
\langle f(a+l(b-a))-f(a), u\rangle=\langle l(f(b)-f(a)), u\rangle .
$$

or there is a $u \in Y^{*}$ such that

$$
\left\langle\lim _{t \rightarrow 0+} \frac{f(a+t(b-a))-f(a)}{t}, u\right\rangle=\langle f(b)-f(a), u\rangle .
$$

We note that if $Y=\mathbb{R}$, then we may choose $u=1$ and $\langle\cdot, \cdot\rangle$ is merely multiplication. In this case, the previous condition reduces to the condition where the graph of $f$ intersects its chord in the extended sense.

We can now state a generalization of Theorem 2.1 for functions defined on topological vector spaces.

THEOREM 2.3. Let $X, Y$ be Hausdorff topological vector spaces over the field $\mathbb{R}$ of real numbers, let $A \subset X$ be an open set and let $Y^{*}$ denote the continuous dual of $Y$. Let $f: A \rightarrow Y$ be a function continuous on the line segment $\left[x_{0}, x_{0}+h\right] \subset$ A. Let $J=\{x \in$ $\left[x_{0}, x_{0}+h\right]: f$ is not Gateaux differentiable at $\left.x\right\}$ and set $j=|J|$. Let

$$
F \ell(v)=\frac{1}{v^{2}}\left[v f^{\prime}\left(x_{0}+v h\right)(h)-\left(f\left(x_{0}+v h\right)-f\left(x_{0}\right)\right)\right] .
$$

(1) If $j=0$, then for each $u \in Y^{*}$ there exists $v \in(0,1)$ such that

$$
\langle F \ell(v), u\rangle=\frac{1}{2}\left\langle f^{\prime}\left(x_{0}+v h\right)(h)-f^{\prime}\left(x_{0}\right)(h), u\right\rangle .
$$

(2) If $j \leq n$ for some nonnegative integer $n$ and $x_{0} \notin J$, then for each $u \in Y^{*}$ there exist $n+1$ points $v_{1}, v_{2}, \ldots, v_{n+1} \in(0,1)$ and $n+1$ positive numbers $\alpha_{1}, \alpha_{2}, \ldots$, $\alpha_{n+1}$ such that

$$
\begin{gathered}
\alpha_{1}+\alpha_{2}+\cdots+\alpha_{n+1}=1 \\
\left\langle\sum_{i=1}^{n+1} \alpha_{i} F \ell\left(v_{i}\right), u\right\rangle=\left\langle\left(f\left(x_{0}+h\right)-f\left(x_{0}\right)\right)-f^{\prime}\left(x_{0}\right)(h), u\right\rangle .
\end{gathered}
$$


(3) If $j$ is unbounded and $f$ intersects its chord in the extended sense for some $u \in Y^{*}$, then there is a $t_{0} \in(0,1)$ and $\delta_{1}, \delta_{2}>0$ such that either

$$
\left\langle F \ell_{1}\left(t_{0}, s\right), u\right\rangle \leq 0 \leq\left\langle F \ell_{2}\left(t_{0}, t\right), u\right\rangle
$$

or

$$
\left\langle F \ell_{2}\left(t_{0}, t\right), u\right\rangle \leq 0 \leq\left\langle F \ell_{1}\left(t_{0}, s\right), u\right\rangle
$$

holds for $0<s \leq \delta_{1}$ and $0<t \leq \delta_{2}$ where

$$
\begin{aligned}
& F \ell_{1}\left(t_{0}, s\right)=\left[t_{0} \frac{f\left(x_{0}+t_{0} h\right)-f\left(x_{0}+\left(t_{0}-s\right) h\right)}{s}-\left(f\left(x_{0}+t_{0} h\right)-f\left(x_{0}\right)\right)\right], \\
& F \ell_{2}\left(t_{0}, t\right)=\left[t_{0} \frac{f\left(x_{0}+\left(t_{0}+t\right) h\right)-f\left(x_{0}+t_{0} h\right)}{t}-\left(f\left(x_{0}+t_{0} h\right)-f\left(x_{0}\right)\right)\right] .
\end{aligned}
$$

Proof. Let $u \in Y^{*}$ and define the function $\phi:[0,1] \rightarrow \mathbb{R}$ by

$$
\phi(t)=\left\langle f\left(x_{0}+t h\right), u\right\rangle .
$$

For each $t$ in $[0,1]$ where $\phi$ is differentiable, we have

$$
\phi^{\prime}(t)=\left\langle f^{\prime}\left(x_{0}+t h\right)(h), u\right\rangle .
$$

If $j=0$, then $\phi$ is differentiable on the entire interval [0,1]. It follows from Theorem 2.1(1) that there exists a $v \in(0,1)$ such that

$$
\frac{1}{2} \frac{\phi^{\prime}(1)-\phi^{\prime}(0)}{1-0} v^{2}=\frac{1}{v^{2}}\left[(v-0) \phi^{\prime}(v)-(\phi(v)-\phi(0))\right] .
$$

Using (2.29) and (2.30) and simplifying yields the first item in Theorem 2.3.

If $j \leq n$ for some nonnegative integer $n$ and $x_{0} \notin J$, then $\phi$ is differentiable on $[0,1]$ except possibly at $n$ points in $(0,1]$. Thus, the second item in Theorem 2.3 follows directly from the second item in Theorem 2.1.

Finally, if $j$ is unbounded and $f$ intersects its chord in the extended sense for some $u \in Y^{*}$, then the graph of $\phi$ intersects its chord in the extended sense and so the third item in Theorem 2.3 follows directly from the third item in Theorem 2.1. This completes the proof.

ACKNOWLEDGEMENT. The authors are grateful to the referee for the thorough review of this paper.

\section{REFERENCES}

[1] R. M. Davitt, R. C. Powers, T. Riedel, and P. K. Sahoo, Flett's mean value theorem for homomorphic functions, Math. Mag. 72 (1999), no. 4, 304-307. MR 2000i:26006.

[2] J. B. Díaz and R. Výborný, On some mean value theorems of the differential calculus, Bull. Austral. Math. Soc. 5 (1971), 227-238. MR 45\#2109. Zbl 216.09302.

[3] T. M. Flett, A mean value theorem, Math. Gaz. 42 (1958), 38-39.

[4] L. A. Khan, Mean value theorem in topological vector spaces, C. R. Math. Rep. Acad. Sci. Canada 19 (1997), no. 1, 24-27. MR 98k:58023. Zbl 896.58007. 
[5] P. K. Sahoo and T. Riedel, Mean Value Theorems and Functional Equations, World Scientific Publishing, New Jersey, 1998. MR 2001h:39027.

ROBERT C. POWERS: DEPARTMENT OF MATHEMATICS, UNIVERSITY OF LOUISVILLE, LOUISVILLE, KY 40292, USA

E-mail address: rcpowe01@athena. 1ouisvi11e.edu

THOMAS RIEDEL: DEPARTMENT OF MATHEMATICS, UNIVERSITY OF LOUISVILLE, LOUISVILLE, KY 40292, USA

E-mail address: thomas.riede1@1ouisvi11e.edu

Prasanna K. SAHOO: Department of Mathematics, University of Louisville, LOUISVILLE, KY 40292, USA

E-mail address: sahoo@1 oui svi11e.edu 


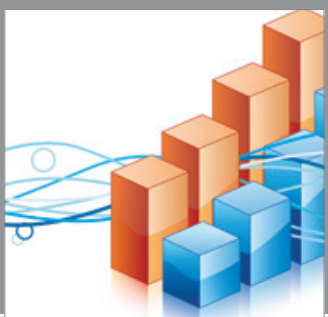

Advances in

Operations Research

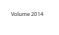

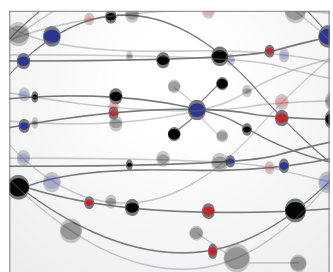

\section{The Scientific} World Journal
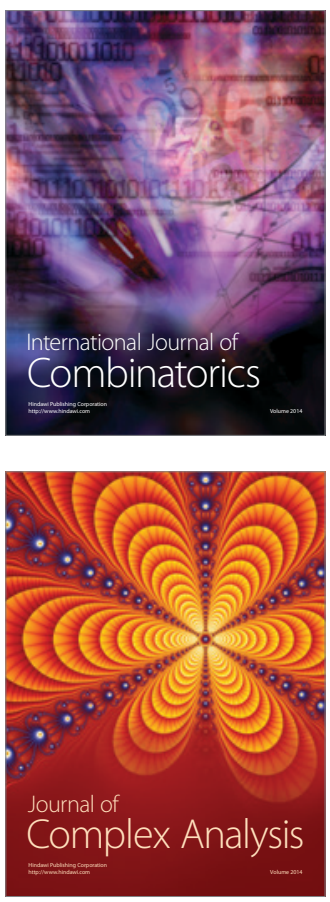

International Journal of

Mathematics and

Mathematical

Sciences
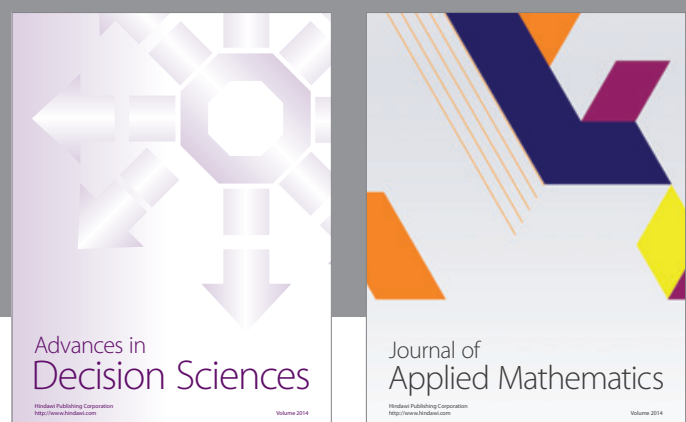

Journal of

Applied Mathematics
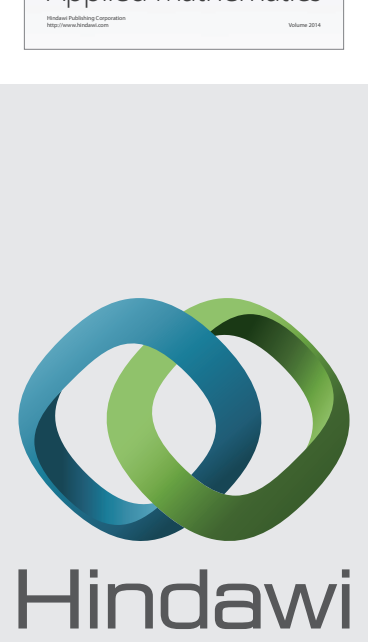

Submit your manuscripts at http://www.hindawi.com
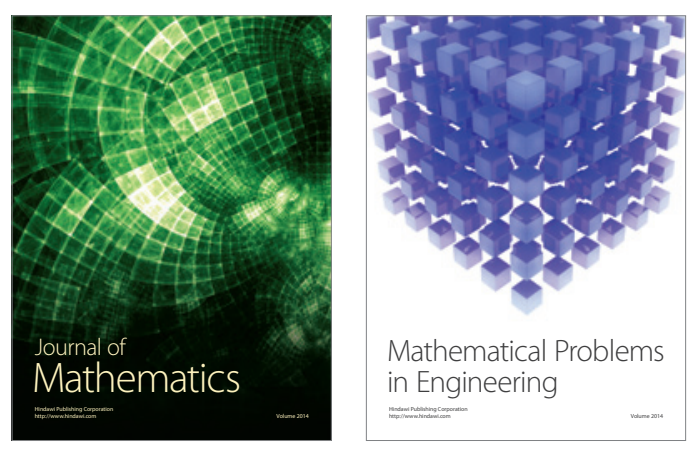

Mathematical Problems in Engineering
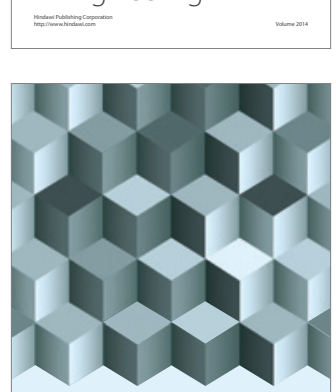

Journal of

Function Spaces
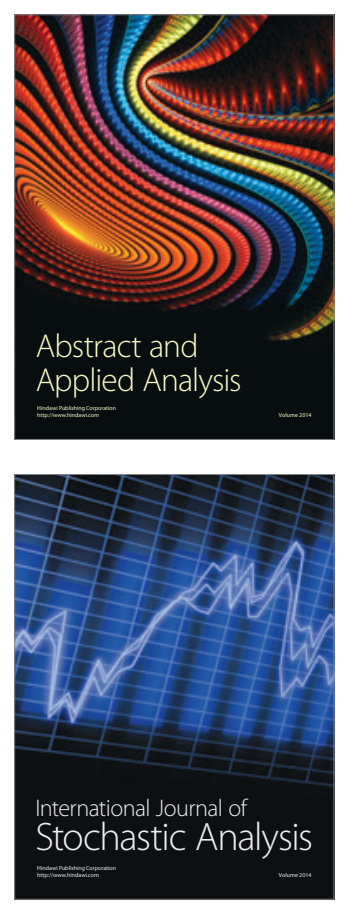

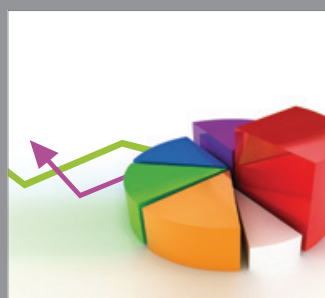

ournal of

Probability and Statistics

Promensencen
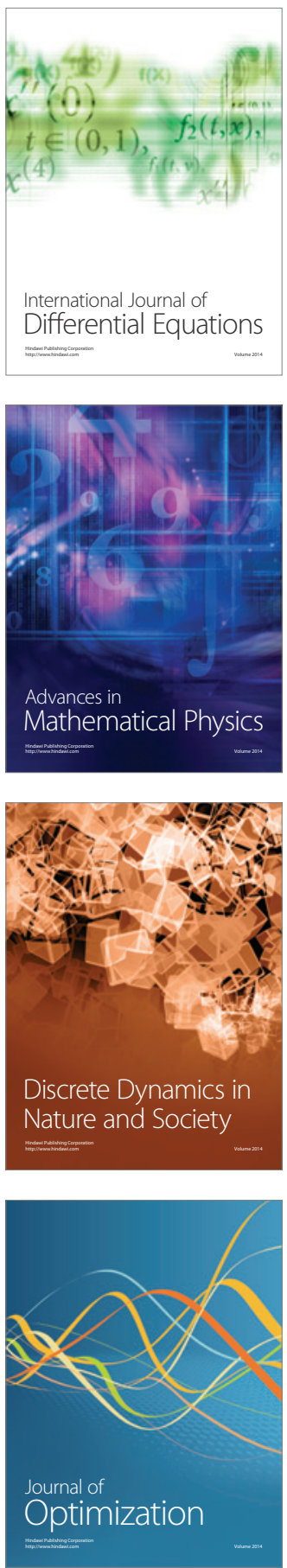